\title{
Peroxiredoxin 6 from cuttlefish (Sepiella maindroni): molecular characterisation during development and the response to thermal stress
}

\author{
Weiwei Song $\cdot$ Chunlin Wang $\cdot$ Ronghua Li $\cdot$ Changkao Mu
}

Received: 2 March 2017/Accepted: 28 August 2017/Published online: 19 September 2017

(C) The Author(s) 2017. This article is an open access publication

\begin{abstract}
The aim of this work was to investigate the function of the peroxiredoxin family member Peroxiredoxin 6 in Sepiella maindroni (Smprx6) during embryonic development and the response to thermal stress. The 1064 bp full-length coding sequence of Smprx6 includes an open reading frame of 660 bp encoding a polypeptide of 219 amino acids with a predicted molecular mass of $24.4 \mathrm{kDa}$. The deduced protein sequence shares significant homology with other reported Prx6 enzymes. Phylogenetic analysis classified SmPrx6 into the 1-Cys-Prx cluster, and quantitative real-time PCR (qPCR) demonstrated constitutive mRNA expression in all tested tissues and during five different embryonic developmental stages, as previously documented in other cephalopods. Thermal stress induced Smprx6 expression, which peaked at $6 \mathrm{~h}$ then returned to control levels. Recombinant SmPrx6 was active at different concentrations and was thiol-dependant. The results suggest that SmPrx6 plays a major role in the development of Sepiella maindroni and its response to oxidative and thermal stress.
\end{abstract}

Keywords Sepiella maindroni · Peroxiredoxin 6 (Prx6) - Embryonic development · Thermal stress · Phylogenetic analysis - qPCR

\section{Introduction}

Cephalopods have high rates of growth and food conversion, which for aquaculture results in a short culture cycle, a high ratio of production to biomass, and high cost-effectiveness. However, at present, only small-scale culturing of a limited number of species is possible (Castellanos-Martínez and Gestal 2013). Furthermore, most reported culturing procedures rely on captured wild individuals (Vidal et al. 2014). Our research group previously developed a system for breeding Sepiella maindroni, a commercially important fishery species accounting for a large proportion of cuttlefish aquaculture in China, by creating artificial culture cycles capable of rearing at least six generations in captivity (Wei-Wei and Chun-Lin 2009). However, despite the

W. Song $\cdot$ C. Wang $(\bowtie) \cdot$ R. Li $\cdot$ C. Mu

Key Laboratory of the Ministry of Education for Applied Marine Biotechnology, School of Marine, Ningbo University, Fenghua Rd. 818, Ningbo 315211, Zhejiang, People's Republic of China

e-mail: wangchunlinnbdx@163.com

W. Song $\cdot$ C. Wang $\cdot$ R. Li $\cdot$ C. Mu

Collaborative Innovation Center for Zhejiang Marine High-Efficiency and Healthy Aquaculture, Ningbo University, Ningbo, People's Republic of China 
development of an artificial rearing method, S. maindroni suffers from the predicament of a high summer mortality. Cephalopods are susceptible to environmental changes, especially water temperature fluctuations, but if they survive such changes, they readily adapt to new environmental conditions (Gestal and CastellanosMartinez 2015). Thermal fluctuations in a marine environment can induce physiological changes through oxidative stress-related mechanisms that generate reactive oxygen species (ROS) to modulate gene expression (Bogdan et al. 2000).

Although low concentrations of ROS are essential for normal physiological signalling (Poynton and Hampton 2014), excess ROS and oxidative stress can lead to cell damage, mutations, and even death (Zheng et al. 2010). Antioxidants are known to play a critical role in regulating ROS levels by detoxifying deleterious oxygen intermediates without disrupting normal cellular functions (Wood et al. 2003b). Peroxiredoxins (Prx) are a ubiquitous family of antioxidant enzymes that prevent oxidative damage (Robinson et al. 2010) and serve as effective medicinal agents for treating ROS-related diseases (Poynton and Hampton 2014). The Prx family has six isoforms classified into two sub-groups, including five 2-Cys-Prxs (Prx1 to Prx5) and one 1-Cys Prx (Prx6). The 2-Cys Prxs are divided into 'typical' and 'atypical' classes, depending on whether the conserved cysteine residues form intermolecular or intramolecular disulfide bridges, respectively (Wood et al. 2003b).

Prx6 is a bifunctional enzyme possessing both peroxidase and phospholipase A2 (PLA2) activities (Lee et al. 2015). Prx6 has been studied in various aquatic organisms including rock cod (Trematomus bernacchii) (Tolomeo et al. 2016), Japanese eel (Anguilla japonica) (Priyathilaka et al. 2016), turbot (Scophthalmus maximus) (Zheng et al. 2010), Chinese mitten crab (Eriocheir sinensis) (Mu et al. 2009), bivalve (Laternula elliptica) (David et al. 2007), disk abalone (Haliotis discus discus) (Nikapitiya et al. 2009), oyster (David et al. 2007), black tiger shrimp (Penaeus monodon) (Qiu et al. 2010) and Chinese shrimp (Fenneropenaeus chinensis) (Zhang et al. 2007). Prx6 has a single conserved catalytic cysteine residue in the $\mathrm{N}$-terminal catalytic motif (PVCTTE) that is responsible for the peroxidase activity (Manevich and Fisher 2005). Evidence suggests Prx6 is distributed in all major tissues that are sensitive to oxidative stress (Manevich and Fisher 2005), and its expression is induced by stimuli such as bacteria (Zheng et al. 2010), viruses (Nikapitiya et al. 2009), pathogen-associated molecular patterns (PAMPs) (De Zoysa et al. 2012), chemicals (Wang et al. 2008), thermal stress (Park et al. 2008; Tolomeo et al. 2016) and environmental pollutants (David et al. 2007).

Antioxidant enzymes operating within the oxidant system of $S$. maindroni are significantly affected by thermal stimulation, particularly those related to scavenging ROS, as demonstrated by cDNA microarray studies based on an EST library (in review). A better understanding of the mechanisms underpinning the responses to oxidative stress could help to improve squid rearing and cephalopod aquaculture. In this study, we identified a peroxiredoxin 6 homolog in S. maindroni (Smprx6), measured Smprx6 mRNA levels in the liver during oxidative and thermal stress, and produced and characterised the recombinant protein.

\section{Materials and methods}

Animals, embryos, acute thermal temperature challenge and sample collection

S. maindroni weighing $158.2 \pm 2.8 \mathrm{~g}$ were collected in Ningbo, China, and acclimated in a blue plastic cylinder tank (diameter $1 \mathrm{~m}$, depth $0.6 \mathrm{~m}$ ) with a salinity of $28-30 \%$ at $22 \pm 2{ }^{\circ} \mathrm{C}$ for 1 week before processing. Cuttlefish $(120$; length $=3.1 \pm 0.5 \mathrm{~cm})$ were randomly divided into six groups containing 20 individuals for the thermal stress experiment. After acclimation for 2 days, three groups were exposed to thermal stress conditions by gradually increasing the temperature by $1{ }^{\circ} \mathrm{C}$ per $\mathrm{h}$ to $32{ }^{\circ} \mathrm{C}$, which is the thermal limit for survival based on breeding experiments (Jiang et al. 2010). Additionally, three control groups were maintained at $22{ }^{\circ} \mathrm{C}$, which is the normal temperature for rearing in seawater. After treatment, five individuals were randomly sampled at $0,0.5,2,6,12,24,48$ and $72 \mathrm{~h}$, and the liver was collected and preserved in RNAlater (TaKaRa, Tokyo, Japan) for RNA extraction. Eight tissues (muscle, ink sac, liver, pancreas, ovary, testis, stomach, and gill) were collected from S. maindroni adults, and total RNA was extracted using RNAiso Plus (TaKaRa, Tokyo, Japan) according to the manufacturer's protocol.

Several clutches of fertilised eggs were collected and acclimated at $20 \pm 1{ }^{\circ} \mathrm{C}$. Embryonic development of S. maindroni can be divided into 12 stages (Jiang et al. 2010), with the early stages combined into five major developmental phases: (I) the zygote stage; (II) the pre-embryonic stage, which occurs over an extremely short 
period and includes cleavage, blastula, and gastrula stages; (III) the organogenesis stage, when the main body parts and organs such as the wrist, eye, heart and ink sac are formed; (IV) the morphological integrity stage, which corresponds to further ongoing development of bodily structures, with the yolk sac still present; (V) the pre-hatching stage, during which the yolk sac disappears in preparation for hatching. Embryos were collected at these five stages and used for RNA extraction.

All aspects of the experiment were conducted according to the guidelines provided by the Ethical Committee of Experimental Animal Care at Ningbo University (Ningbo, Zhejiang, China).

Identification and cloning of the full-length cDNA of SmPrx6

A squid cDNA library was constructed from the ink sac using the Creator Smart cDNA Library Construction Kit (TaKaRa, Tokyo, Japan). cDNA clones were selected randomly from the cDNA library, and single-pass sequenced using a random T3 primer on an ABI 3730 Genetic Analyzer (Applied Biosystems, Foster City, USA). A large-scale expressed sequence tag (EST) sequencing project for S. maindroni was initiated to identify and functionally annotate as many unique transcripts as possible. BLAST searches were performed to compare processed cDNA sequences against ESTs and genes available in the GenBank database (http://www. ncbi.nlm.nih.gov/blast) (Wei-Wei et al. 2012). Intriguingly, EST GT618012 (Contig 485; length = 982 bp) was homologous to Prx6 in Rattus norvegicus (RNO17295).

Amplification of flanking sequences was carried out using a 5'- and 3'-Full RACE kit (TaKaRa, Tokyo, Japan) to obtain the complete sequence of EST GT618028 following the manufacturer's instructions. Purified genes were cloned into the pMD-18T vector (Takara, Tokyo, Japan) and transformed into Escherichia coli Top10F-competent cells. Finally, M13-47 and RV-M universal primers (Table 1) were used to screen for three positive recombinants that were subsequently sequenced.

Sequence analysis, multiple sequence alignment and phylogenetic analysis

The sequence similarity of nucleotide and protein sequences was compared using BLAST (http://www.ncbi. nlm.gov/blast). Amino acid sequences were deduced by the Expert Protein Analysis System (http://www. expasy.org). Protein motif features were predicted by the Simple Modular Architecture Research Tool (http:// smart.emblheidelberg.de/) (Schultz et al. 1998). Signal peptide prediction was performed by SignalP 4.1 (http://www.cbs.dtu.dk/services/SignalP). Multiple sequence alignment of Smprx6 was performed using ClustalW (http://www.ebi.ac.uk/clustalw/). The neighbour-joining method was used to perform multiple sequence alignment of Smprx6 and its homologs in other species and construct a phylogenetic network with

Table 1 Oligonucleotide primers used in this experiment

\begin{tabular}{lll}
\hline Primer & Sequence $\left(5^{\prime}-3^{\prime}\right)$ & Sequence information \\
\hline P1 (reverse) & AACTGCACGCGCGGTTTTCA & SmPrx6-specific primer \\
P2 (forward) & GTAAGAATGCCAGTCGTGTT & SmPrx6-specific primer \\
P3 (reverse) & CATGGCTACATGCTGACAGCCTA & Universal primer \\
P4 (forward) & TACCGTCGTTCCACTAGTGATTT & Universal primer \\
P5 (reverse) & ACTGAGCATCCTCTATCCAG & Real-time SmPrx6 primer \\
P6 (forward) & TATCACCAACCTTCCAGTCG & Real-time SmPrx6 primer \\
P7 (reverse) & GACTCCTACGTAGGAGACGA & Real-time $\beta$-actin primer \\
P8 (forward) & CGTTGAAGGTCTCGAACATGA & Real-time $\beta$-actin primer \\
P7 (reverse) & CATATGGCTAACCTTGGAGATATTTTCCCA & Recombinant primer (for expression) \\
P8 (forward) & CTCGAGTTAGTGGTGGTGGTGGTGGTGTTCTGGA & Recombinant primer (for expression) \\
T3 & TGGGGTGTCATACGA & \\
T7 & AATTAACCCTCACTAAAGGG & Vector primer \\
M13-47 & GTAATACGACTCACTATAGGGC & Vector primer \\
RV-M & CGCCAGGGTT TTCCCAGTCACGAC & Vector primer \\
\hline
\end{tabular}


MEGA 6.0. Tertiary structures of Smprx6 were predicted using the SWISSMODEL prediction algorithm (http://swissmodel.expasy.org).

Transcriptional analysis of SmPrx6 by quantitative real-time RT-PCR

Quantitative real-time RT-PCR was performed using an ABI 7300 (Applied Biosystems, Foster City, USA). Reactions were carried out in a final volume of $25 \mu \mathrm{L}$ containing $12.5 \mu \mathrm{L}$ of SYBR Green Real-time PCR Master Mix (TaKaRa, Tokyo, Japan), $9.5 \mu \mathrm{L}$ of water, $0.5 \mu \mathrm{L}$ of each primer (P5 and P6 for SmPrx6, and P7 and $\mathrm{P} 8$ for $\beta$-actin; see Table 1), and $2 \mu \mathrm{L}$ of cDNA. Reactions were incubated at $95{ }^{\circ} \mathrm{C}$ for $3 \mathrm{~s}$, followed by 40 cycles at $95{ }^{\circ} \mathrm{C}$ for $5 \mathrm{~s}, 56{ }^{\circ} \mathrm{C}$ for $5 \mathrm{~s}$, and $72{ }^{\circ} \mathrm{C}$ for $20 \mathrm{~s}$, and a final extension was performed at $72{ }^{\circ} \mathrm{C}$ for $1 \mathrm{~min}$. Reactions were performed in a 96-well plate, each sample was repeated in triplicate, and an internal control gene ( $\beta$-actin) was included. Dissociation curve analysis of amplification products was performed at the end of each reaction to confirm that only one PCR product was amplified and detected. PCR data were analysed using the ABI 7300 SDS software (Applied Biosystems, Foster City, USA). To maintain consistency, the baseline was set automatically by the software. The comparative $C_{\mathrm{t}}$ method was used to analyse the relative expression levels of Smprx6. $C_{\mathrm{t}}$ values for the amplified target products and the internal control ( $\beta$ actin) were determined for each sample, and the difference in $C_{\mathrm{t}}$ for the target and internal control was calculated to normalise the differences in template and the efficiency of RT-PCR. Liver from squid in the control group was used as the reference sample for calibration. $\Delta C_{\mathrm{t}}$ values for each sample were subtracted from the $\Delta C_{\mathrm{t}}$ of the calibrator to generate $\Delta \Delta C_{\mathrm{t}}$ values. The expression level of Smprx6 was calculated using the $2^{-\Delta \Delta C \mathrm{t}}$ method, and values represent the $\mathrm{n}$-fold difference compared with the calibrator (Livak and Schmittgen 2001).

Expression of recombinant SmPrx6

A PCR fragment encoding the mature SmPrx6 polypeptide was amplified with gene-specific primers P7 and P8 (Table 1) and cloned into the pMD18-T simple vector (TaKaRa, Tokyo, Japan), digested with restriction enzymes NdeI and XhoI (New England Biolabs, MA, USA), then subcloned into the NdeI and XhoI sites of the expression vector pET-21a(+) (Novagen, Shanghai, China). The recombinant plasmid was transformed into E. coli Origami (DE3) (Novagen, Shanghai, China) and subjected to DNA sequencing. The parent vector without the inserted fragment was used as a negative control. After sequencing to confirm in-frame insertion, positive transformants and the negative control were incubated in LB medium containing $50 \mu \mathrm{g} / \mathrm{mL}$ ampicillin at $37{ }^{\circ} \mathrm{C}$ with shaking at $220 \mathrm{rpm}$. When the culture reached an $\mathrm{OD}_{600}$ of $0.5-0.7$, IPTG was added to a final concentration of $1 \mathrm{mM}$ and culturing continued for an additional $4 \mathrm{~h}$ under the same conditions.

Cells were harvested by centrifugation at $8000 \times g$ for $2 \mathrm{~min}$ and suspended in $50 \mathrm{mM}$ Tris containing $5 \mathrm{mM}$ EDTA, $50 \mathrm{mM} \mathrm{NaCl}$, and 5\% glycerol (pH 7.9). After sonication for $60 \mathrm{~s}$ on ice, the rSmPrx6 and negative control samples were purified using a HisTrap Chelating Column (Amersham Biosciences, Stockholm, Sweden) according to the manufacturer's instructions. Purified recombinant proteins were analysed by 15\% SDS-PAGE to check the molecular mass and purity. Assaying of the antioxidant activity was performed using the Total Antioxidant Capacity Assay Kit (Jiancheng Bioengineering Institute, Nanjing, China) using the 2,2'-azino-bis(3-ethyl-benzothiazoline-6-sulphonic acid) ABTS method (Miller et al. 1993). Peroxidase activity was measured based on the change in absorbance at $420 \mathrm{~nm}$ that accompanies the breakdown of $\mathrm{H}_{2} \mathrm{O}_{2}$. One unit of activity was defined as the amount of enzyme catalysing the breakdown of $1 \mathrm{mg}$ of substrate by $1 \mathrm{~g}$ of fresh tissue in the reaction system at $37^{\circ} \mathrm{C}$. Peroxidase activity was calculated using the formula supplied with the Peroxidase assay kit (Jiancheng Bioengineering Institute, Nanjing, China).

Statistical analysis

All data were expressed as the mean \pm standard deviation $(n=5)$, and mean values were subjected to oneway analysis of variance (ANOVA). SPSS20 software was used to determine statistical significance $(p<0.05)$, and Origin 8.0 was used to prepare figures. 


\section{Results}

Cloning and analysis of SmPrx6

The complete cDNA sequence of Smprx6 was obtained by overlapping EST GT618012 with amplified fragments. The sequence has been deposited in Genbank under accession number HQ662844. The full-length mRNA of Smprx6 is $1064 \mathrm{bp}$ and includes an open reading frame of $660 \mathrm{bp}$ encoding a polypeptide of 219 amino acids with a predicted molecular mass of $24.4 \mathrm{kDa}$ and a theoretical isoelectric point of 6.17 (Fig. 1). The deduced amino acid sequence of Smprx6 is shown in Fig. 1. The protein includes a typical Prx domain (from Leu4 to Ser160) and an alkyl hydroperoxide reductase (AhpC) domain (from Leu4 to Ser140) as identified by the SMART program. A signal peptide was not predicted by SignalP.

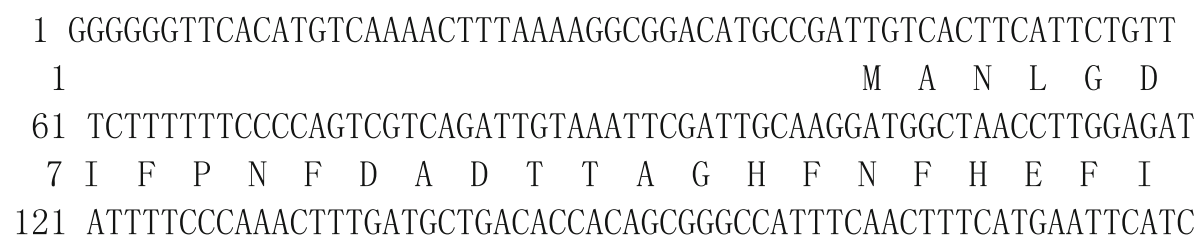

$\begin{array}{llllllllllllllllllllll}27 & \mathrm{~N} & \mathrm{~N} & \mathrm{~S} & \mathrm{~W} & \mathrm{~A} & \mathrm{I} & \mathrm{L} & \mathrm{F} & \mathrm{S} & \mathrm{H} & \mathrm{P} & \mathrm{A} & \mathrm{D} & \mathrm{Y} & \mathrm{T} & \mathrm{P} & \mathrm{V} & \mathrm{C} & \mathrm{T} & \mathrm{T}\end{array}$

181 AATAATTCTTGGGCCATCCTCTTCTCTCATCCTGCTGATTACACACCTGTCTGCACAACG $\begin{array}{llllllllllllllllllllll}47 & \mathrm{E} & \mathrm{L} & \mathrm{T} & \mathrm{R} & \mathrm{V} & \mathrm{V} & \mathrm{Q} & \mathrm{L} & \mathrm{I} & \mathrm{P} & \mathrm{E} & \mathrm{F} & \mathrm{K} & \mathrm{K} & \mathrm{R} & \mathrm{G} & \mathrm{V} & \mathrm{K} & \mathrm{L} & \mathrm{C}\end{array}$ 241 GAGCTGACGAGAGTTGTCCAGTTGATTCCAGAATTCAAGAAAAGGGGAGTGAAACTGTGT $\begin{array}{lllllllllllllllllllll}67 & \text { A } & \text { L } & \text { S } & \text { C } & \text { D } & \text { D } & \text { V } & \text { E } & \text { S } & \text { H } & \text { H } & \text { G } & \text { W } & \text { I } & \text { K } & \text { D } & \text { I } & \text { I } & \text { A } & \text { Y }\end{array}$ 301 GCCTTGTCATGTGATGATGTTGAAAGTCATCATGGTTGGATAAAAGATATTATTGCTTAC $\begin{array}{lllllllllllllllllllll}87 & G & K & \text { L } & \text { A } & \text { E } & \text { F } & \text { P } & \text { Y } & \text { P } & \text { I } & \text { I } & \text { S } & \text { D } & \text { K } & \text { T } & \text { R } & \text { K } & \text { L } & \text { A } & \text { V }\end{array}$ 361 GGCAAACTTGCTGAGTTTCCATATCCAATCATCTCTGACAAAACCCGAAAGCTCGCAGTC $\begin{array}{lllllllllllllllllllllll}107 & \mathrm{~S} & \mathrm{~L} & \mathrm{G} & \mathrm{M} & \mathrm{V} & \mathrm{D} & \mathrm{P} & \mathrm{D} & \mathrm{E} & \mathrm{K} & \mathrm{D} & \mathrm{L} & \mathrm{A} & \mathrm{G} & \mathrm{M} & \mathrm{P} & \mathrm{L} & \mathrm{T} & \mathrm{A} & \mathrm{R}\end{array}$ 421 AGTCTGGGTATGGTGGATCCTGATGAAAAGGACCTGGCTGGAATGCCCCTAACTGCACGC $\begin{array}{lllllllllllllllllllll}127 & \mathrm{~A} & \mathrm{~V} & \mathrm{~F} & \mathrm{I} & \mathrm{I} & \mathrm{G} & \mathrm{P} & \mathrm{D} & \mathrm{K} & \mathrm{K} & \mathrm{L} & \mathrm{K} & \mathrm{L} & \mathrm{S} & \mathrm{I} & \mathrm{L} & \mathrm{Y} & \mathrm{P} & \mathrm{A} & \mathrm{T}\end{array}$ 481 GCGGTTTTCATCATTGGCCCTGACAAGAAATTAAAACTGAGCATCCTCTATCCAGCAACC $\begin{array}{lllllllllllllllllllll}147 & \mathrm{~T} & \mathrm{G} & \mathrm{R} & \mathrm{N} & \mathrm{F} & \mathrm{D} & \mathrm{E} & \mathrm{I} & \mathrm{L} & \mathrm{R} & \mathrm{V} & \mathrm{I} & \mathrm{D} & \mathrm{S} & \mathrm{L} & \mathrm{Q} & \mathrm{L} & \mathrm{T} & \mathrm{A} & \mathrm{K}\end{array}$ 541 ACTGGGAGAAATTTTGATGAAATTTTGAGAGTGATTGATTCCCTCCAATTAACGGCTAAG $\begin{array}{lllllllllllllllllllll}167 & \mathrm{H} & \mathrm{K} & \mathrm{V} & \text { A } & \mathrm{T} & \mathrm{P} & \mathrm{V} & \mathrm{D} & \text { W } & \mathrm{K} & \mathrm{V} & \mathrm{G} & \mathrm{D} & \mathrm{K} & \mathrm{C} & \mathrm{M} & \mathrm{V} & \mathrm{V} & \mathrm{P} & \mathrm{S}\end{array}$ 601 CACAAGGTGGCCACTCCTGTCGACTGGAAGGTTGGTGATAAATGCATGGTTGTCCCCTCC

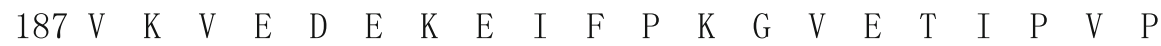
661 GTCAAAGTTGAAGACGAAAAAGAAATCTTCCCCAAGGGGGTTGAAACAATCCCAGTGCCT $\begin{array}{llllllllllllllllll}207 & \mathrm{~S} & \mathrm{G} & \mathrm{K} & \mathrm{S} & \mathrm{Y} & \mathrm{L} & \mathrm{R} & \mathrm{M} & \mathrm{T} & \mathrm{P} & \mathrm{H} & \mathrm{P} & \mathrm{E} & *\end{array}$ 721 TCAGGAAAATCCTATCTTCGTATGACACCCCATCCAGAATAAAGAAATGCAGAAAGGGCA 781 GGACTGCTGACCTTTTTTGCTTTTGACCTCTTTGAGATAAATTCAAAGGTGGGGGGGCAG 841 GGGGCTACTCTAAGAACAAAATACTGCTGTAATTAATTGTAAGAATGCCAGTCGTGTTTG 901 AAATCTATTTTTTTTTTCTACTCATAACGAATTGTTTAAAATCTGATTGTATAAATCATT 961 TTGAAAGTAAAAACAGTTTTGTATTTGAGTCGTTCTACCGACTGTAATTTGGATGAAGGG 1021 ATGAATTTGATTTCAATAAAGATTTCTAAAGGAAAAAAAAAAAA

Fig. 1 Nucleotide and deduced amino acid sequences of SmPrx6. The asterisk (*) indicates the stop codon. The polyadenylation signal is shown in bold italics. The active cysteine residue is in bold and is underlined. The catalytic motif is shaded. The conserved positively charged His 36 and Arg 126 are enclosed by solid lines 
Multiple sequence alignment and phylogenetic analysis

The deduced amino acid sequence of Smprx6 shares significant homology with other reported Prx6 proteins, with $71 \%$ identity with Saccostrea glomerata (ACX50969), and 70.9\% identify with Haliotis discus discus (ABO26614). Multiple alignments revealed that Cys44 is conserved in all analysed Prx VI sequences. Moreover, the predicted SmPrdx6 protein has a unique cysteine residue (C44) in the peroxidase motif (42PVCTTE47) and a catalytic serine residue (S29) in the lipase motif (27NNSWA31) at the N-terminus (Fig. 2). Both of them are essential amino acid residues in the active sites that mediate peroxidase and PLA2 activity, respectively.

A phylogenetic tree was constructed based on Prx amino acid sequences (Fig. 3). Three distinct groups (1Cys, 2-Cys, and an atypical 2-Cys subgroup) formed distinct clusters, with all Prx5 proteins part of the atypical 2-Cys subgroup, and Prx1, Prx2, Prx3, and Prx4 clustered in the 2-Cys subgroup. The Smprx6 sequence and other Prx6 sequences from invertebrates were placed in the 1-Cys subgroup.

Expression of Smprx6 in different tissues

To determine tissue-specific mRNA expression profiles of Smprx6, quantitative RT-PCR was carried out using various tissues from unchallenged (control) cuttlefish. Expression levels were normalised against $\beta$-actin mRNA levels which were tested in different tissues and following treatments to provide an internal control. The results revealed no significant differences in cycle threshold $\left(C_{\mathrm{t}}\right)$ values for $\beta$-actin in different experiments (Fig. $4 ; p>0.05$ ). The expression level of Smprx6 in the ink sac was defined as 1 , and expression was 460.71-fold higher in the pancreas, followed by liver (148.76-fold), gill (90.15-fold), ovary (26.6-fold), testis (11.53-fold), muscle (7.10-fold), and stomach (1.01-fold).

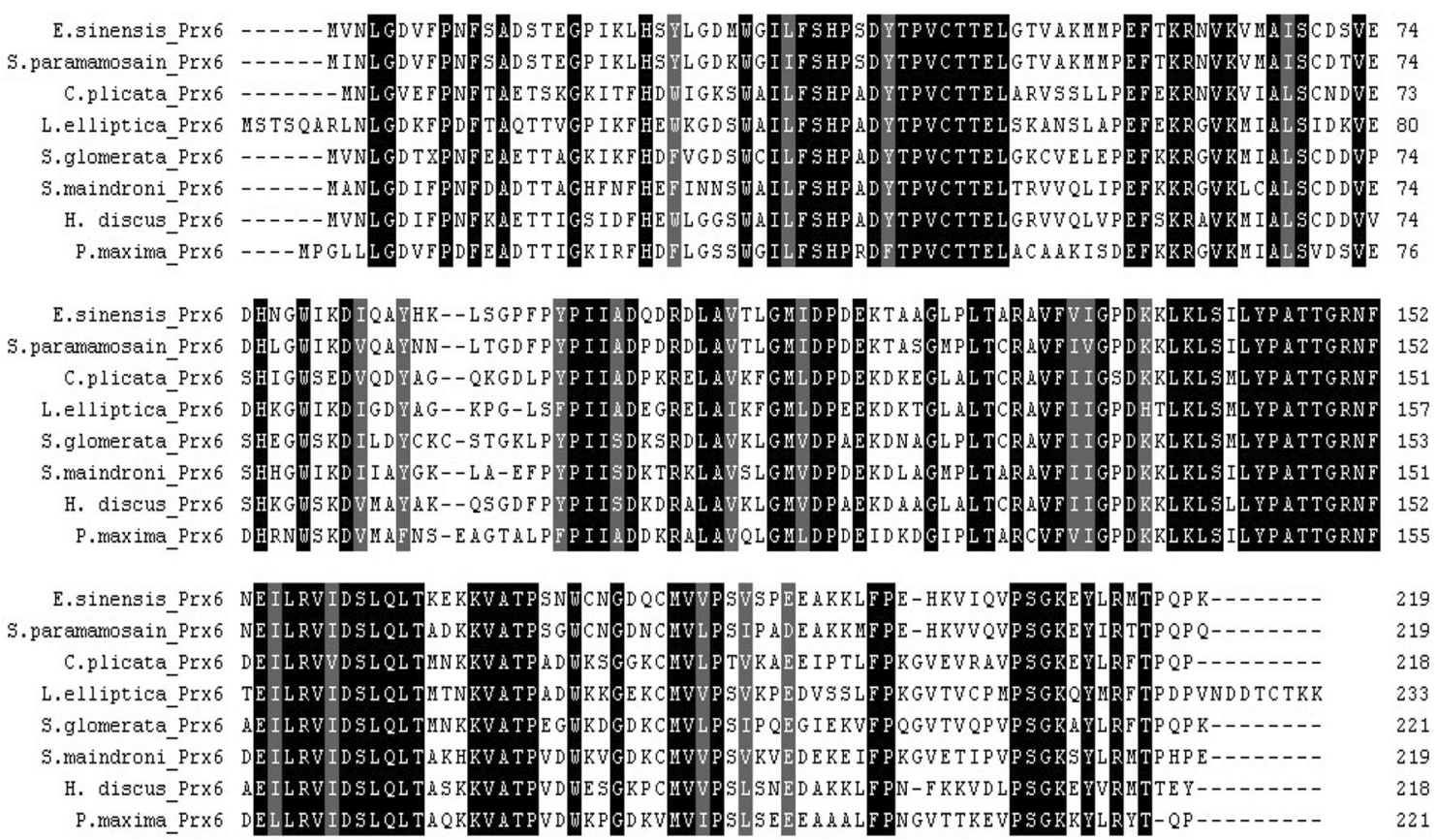

Fig. 2 Multiple alignments of the deduced amino acid sequence of SmPrx6 with other Prx6 proteins deposited in GenBank. Black shading indicates conserved amino acid residues. Gaps are indicated by dashes to improve the alignment. Abbreviations are as follows ACX50969, Saccostrea glomerata_Prx6; ABO26614, Haliotis discus discus_Prx6; ACF35639, Eriocheir sinensis_Prx6; ADN06076, Cristaria plicata_Prx6; ACJ53746, Scylla paramamosain_Prx6; ADJ57694, Psetta maxima_Prx6; ACE76885, Laternula elliptica_Prx6. Prx6 from S. maindroni is designated as Smprx6 


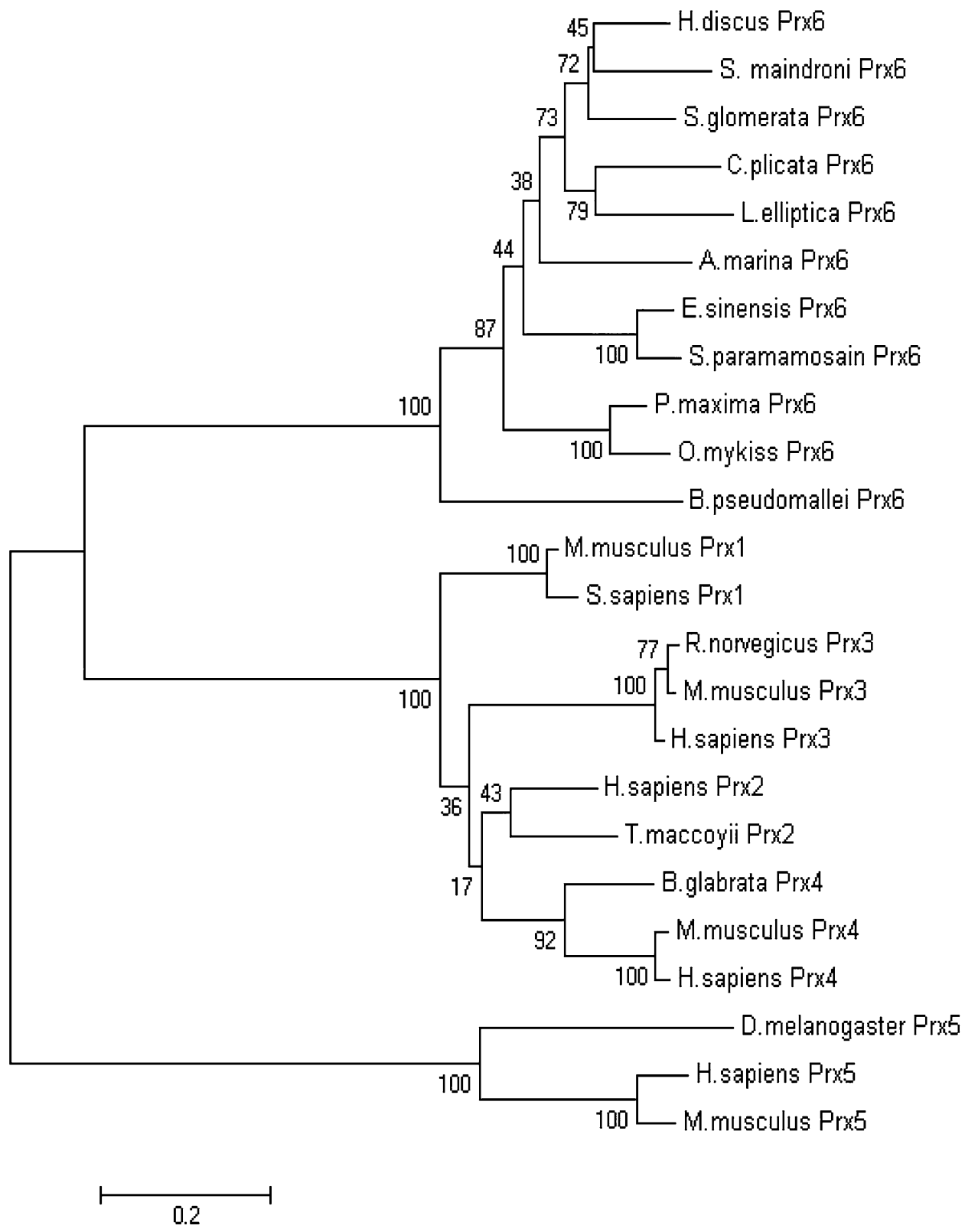

Fig. 3 Consensus neighbour-joining phylogenetic tree based on Prx sequences from the following species: CAM16508, Mus musculus_Prx1; NP_859048, Homo sapiens_Prx1; NP_005800, Homo sapiens_Prx2; ABW88997, Thunnus maccoyii_Prx2; AAH60567, Rattus norvegicus_Prx3; AAH05626, Mus musculus_Prx3; CAG29340, Homo sapiens_Prx3; NP_058044, Mus musculus_Prx4; ACI42880, Biomphalaria glabrata_Prx4; CAG46506, Homo sapiens_Prx4; AAF55497, Drosophila melanogaster_Prx5; CAG33484, Homo sapiens_Prx5; AAG13450, Mus musculus_Prx6; ACX50969, Saccostrea glomerata_Prx6; ABO26614, Haliotis discus_Prx6; ACF35639, Eriocheir sinensis_Prx6; ADN06076, Cristaria plicata_Prx6; AAY96294, Arenicola marina_Prx6; ACJ53746, Scylla paramamosain_Prx6; ADJ57694, Psetta maxima_Prx6; NP_001158604, Oncorhynchus mykiss_Prx6; ACE76885, Laternula elliptica_Prx6

Expression of Smprx6 during embryonic development

Smprx6 mRNA levels in embryos were measured by quantitative RT-PCR and transcripts were detected in all stages of development, with maximum expression during the pre-embryonic stage (1369.11-fold), but expression then decreased markedly during the organogenesis stage (15.2-fold), and only trace amounts were detected in the pre-hatching stage $(p<0.05$; Fig. 5). 


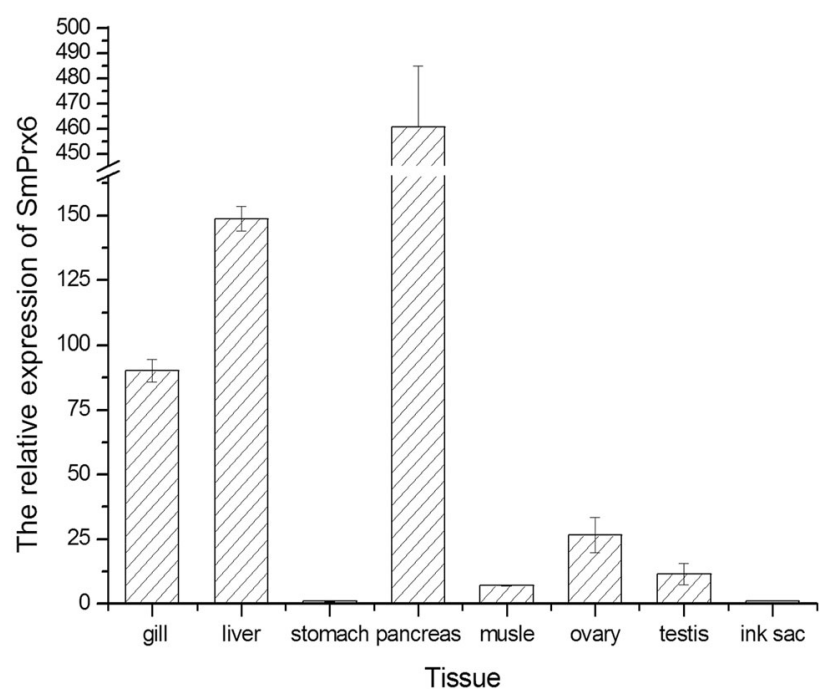

Fig. 4 Tissue distribution of Smprx6 transcripts measured by SYBR Green RT-PCR. Muscle, ink sac, liver, pancreas, ovary, testis, gastric and gill were collected from three individual cuttlefish. Vertical bars represent the mean $\pm \operatorname{SD}(p<0.05)$

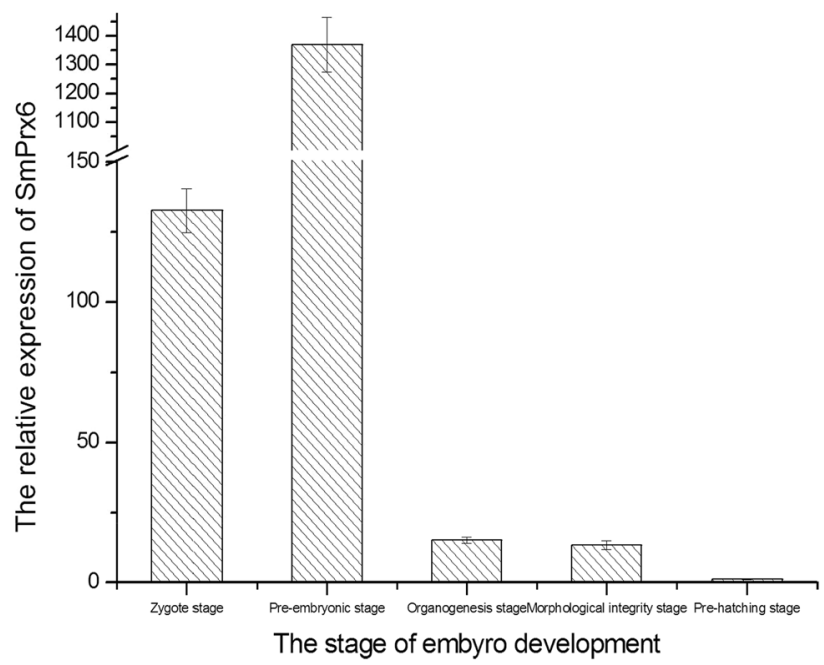

Fig. 5 Smprx6 mRNA expression in embryos measured by SYBR Green RT-PCR. The $\beta$-actin gene was used as an internal control for all samples. Vertical bars represent the mean $\pm \mathrm{SD}$, and bars with different letters are significantly different $(p<0.05)$

Expression of SmPrx6 in liver after thermal treatment

To investigate Smprx6 expression following an external temperature stimulus, S. maindroni were exposed to $32{ }^{\circ} \mathrm{C}$ for $0.5,2,6,12,24,48$ and $72 \mathrm{~h}$, while controls were maintained at $22{ }^{\circ} \mathrm{C}$. Expression of Smprx6 was assayed by quantitative real-time RT-PCR with $\beta$-actin as an internal control. At $1 \mathrm{~h}$ after thermal stimulation, Smprx6 mRNA levels were slightly up-regulated, but the difference from the control group was not significant $(p>0.05)$. Smprx6 mRNA levels then increased rapidly and peaked at $6 \mathrm{~h}$, representing an increase of 3.72fold ( $\mathrm{p}<0.05$ ), before declining and eventually returning to control levels (Fig. 6).

Expression, purification and characterisation of recombinant SmPrx6

The recombinant plasmid pET-21a-SmPrx6 was transformed into E. coli Origami (DE3) as described above, and $\mathrm{rSm} \operatorname{Prx} 6$ was expressed and purified under native conditions. SDS-PAGE confirmed the presence of an overexpressed protein in the whole cell lysate following IPTG induction, and the purified protein ran as a 


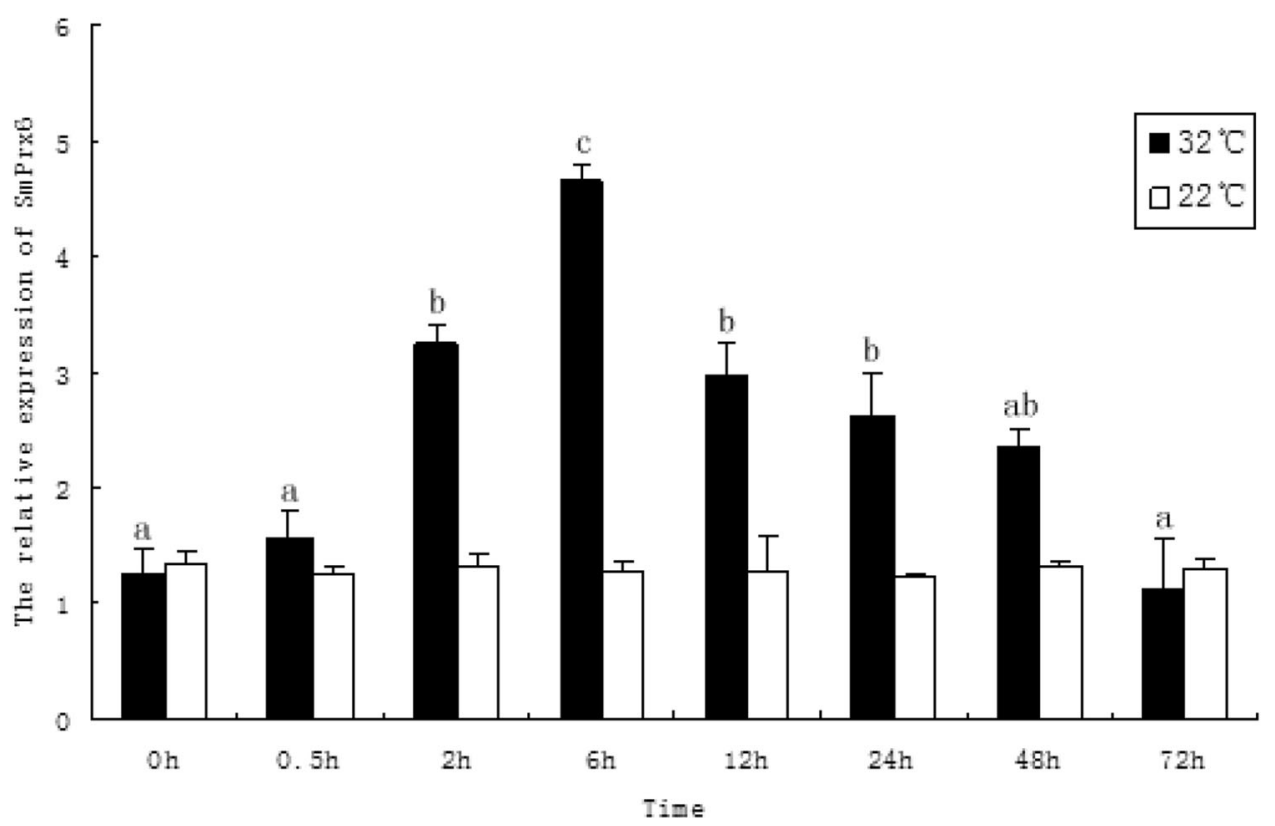

Fig. 6 Temporal expression profile of Smprx6 transcripts in liver following thermal stress measured by SYBR Green RT-PCR. $S$. maindroni adults were incubated at $32{ }^{\circ} \mathrm{C}$ for $0,0.5,2,6,12,24,48$ and $72 \mathrm{~h}$. The control group was maintained at $22{ }^{\circ} \mathrm{C}$. Vertical bars represent the mean $\pm \mathrm{SD}$, and bars with different letters are significantly different $(p<0.05)$

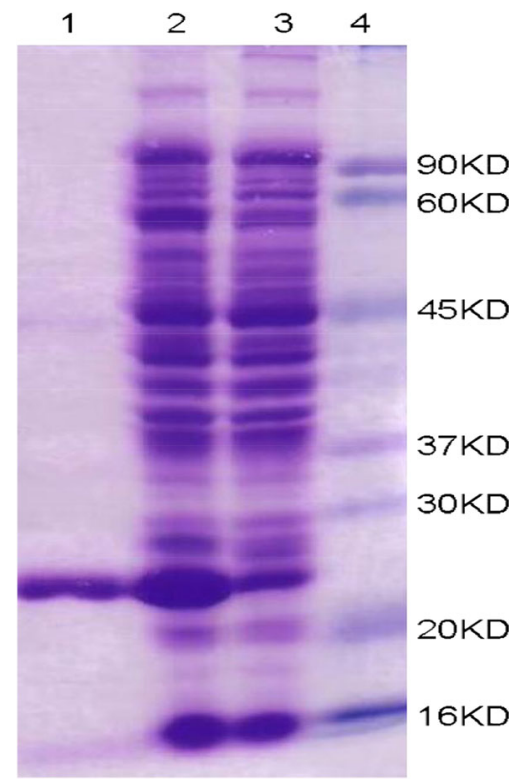

Fig. 7 SDS-PAGE of SmPrx6. Lane 1 purified rSmPrx6; lane 2 expression of rSmPrx6 after IPTG induction; lane 3 uninduced control without IPTG induction; lane 4 protein molecular standards

signal band with a molecular mass ( $\sim 25 \mathrm{kDa}$ ) comparable to that predicted for $\mathrm{rSm} \operatorname{Prx} 6$ (Fig. 7). The purified rSmPrx6 enzyme displayed antioxidant activity of $6.17 \mathrm{U} / \mathrm{mg}$ and POD activity of $10.17 \mathrm{U} / \mathrm{mg}$.

\section{Discussion}

SmPrx6 shares common structural features with other invertebrate and vertebrate Prx6 proteins. The phylogenetic tree showed that Smprx6 falls into the 1-cys-Prx cluster, members of which contain a consensus 
sequence with a conserved cysteine residue (Cys 44) in the $\mathrm{N}$ terminus, and a AhpC domain with a peroxidase catalytic motif (PVCTT). In mammals, Cys47, His39 and Arg132 form the catalytic triad responsible for peroxidase activity, with Cys47 bound to His39 via hydrogen bonding, and electrostatically activated by Arg132 (Manevich and Fisher 2005). Interestingly, a catalytic triad (Cys44, His36 and Arg 126) is also present in squid and other mollusks (Nikapitiya et al. 2009). The catalytic motif responsible for PLA2 activity (27NNSWA31) was observed in the deduced amino acid sequence, with Ser29 the catalytic nucleophile forming part of the NNSWG consensus sequence responsible for the PLA2 hydrolase activity (Lee et al. 2015). Ser is replaced with a Met, Arg or Gly in E. sinensis (Mu et al. 2009), Xenopus laevis (Genbank accession no. NP_001084316) and Suberites domuncula (Genbank accession no. CAC38779), respectively. The effect this replacement has on the catalytic activity warrants further study.

Smprx6 was constitutively transcribed in all examined tissues, suggesting it is ubiquitously expressed in squid, and levels were highest in pancreas and liver, which is different from other aquatic animals. Ploch et al. (1999) reported that liver cells from a variety of fish species are relatively resistant to DNA modification by ROS generated by various compounds. Based on the observed high Prx6 expression levels in pancreas and liver, we propose that Prx6 in squid possesses other activities such as the PLA2 activity described above. Indeed, ubiquitous expression of EsPrx6 mRNA in different tissues has been linked to an involvement in numerous physiological functions including basal metabolism in E. sinensis (Mu et al. 2009). In channel catfish, Prx6 is expressed in liver, head, kidney, intestine, and skin, but not spleen (Yeh and Klesius 2007). However, Zheng and colleagues reported that turbot Prx6 is more highly expressed in blood and heart than liver in unchallenged fish, in contrast with rock bream and channel catfish that display tissue-specific expression profiles (Zheng et al. 2010). This apparent variation in the transcription of Prx6 in different organisms may be due to the expression of multiple isoforms of Prx family members in different organs (Huang et al. 2011).

The consumption of yolk stores during a specific period of development involves governing regulatory signals and controlling ROS by-products (Shafer et al. 2011). Most antioxidant enzymes such as catalase and glutathione peroxidase are expressed at high levels during yolk consumption. Understanding the sub-cellular management of metabolism before, during, and after yolk consumption is, therefore, an intriguing area of investigation. As observed in many other organisms, SmPrx6 mRNA was detected throughout embryonic development. Expression was highest during the pre-embryonic stage (II), which was 1358.26-fold higher than the pre-hatching stage $(p<0.05)$, and expression then declined gradually from stage III to stage V. The early embryonic period can be considered a time of substantial proliferative stress because early-stage embryogenesis involves massive cellular proliferation over a very short time. Correspondingly, SmPrx6 has been implicated in complex cellular processes including cell proliferation (Wood et al. 2003a), differentiation (Rabilloud et al. 1995) and apoptosis (Zhou et al. 2000; Bernier-Villamor et al. 2004), with the highest expression in early-stage embryos. Our present results indicate that SmPrx6 plays a major role in embryonic development in $S$. maindroni, especially during the early stages of tissue differentiation.

Oxidative stress often accompanies thermal stress. Although low levels of ROS modulate a variety of cellular functions such as cell proliferation, high concentrations of ROS cause cell damage or apoptosis (Park et al. 2008). Among the antioxidant systems that protect organisms from excessive ROS, the Prx family of antioxidant enzymes has received much attention during the past decade. The significant upregulation of Prx transcript levels after a high-temperature stimulus indicates the induction of gene expression (Park et al. 2005). Expression of SmPrx6 increased rapidly in S. maindroni and peaked at $6 \mathrm{~h}$ post-stimulus (3.72-fold; $p<0.05$ ) before declining and eventually returning to control levels. This indicates that SmPrx6 may play a major role as an antioxidant protein to protect organisms from excessive ROS following thermal stress (Tolomeo et al. 2016).

Cephalopods are highly adaptable marine organisms, and can adjust their biology and life cycles in response to the prevailing environmental and ecosystem conditions. These organisms can presumably avoid increased levels of oxidative damage normally during short-term thermal stress, possibly by altering metabolic processes.

In conclusion, we identified and characterised Prx6 from S. maindroni, and detected Smprx6 mRNA transcripts in all tested tissues and developmental stages. The observed upregulation of Prx6 expression following a high-temperature stimulus indicates that its peroxidase activity may be important for the response 
to oxidative stress. Therefore, Prx6 may play a major role in maintaining proteins in a reduced state by scavenging toxic ROS.

Acknowledgements The authors are grateful to all the laboratory members for their continuous technical advice and helpful discussion. This research was supported by the National Natural Science Foundation of China [Grant numbers 41176124, 41206114], the Public Welfare Project of Zhejiang Province [Grant number ZX2013000403], the Ningbo Agricultural Major Project [Grant number 201401C1111001], the Open Fund of Zhejiang Provincial Top Key Discipline of Aquaculture at Ningbo University, and the K. C. Wong Magana Fund of Ningbo University.

Open Access This article is distributed under the terms of the Creative Commons Attribution 4.0 International License (http:// creativecommons.org/licenses/by/4.0/), which permits unrestricted use, distribution, and reproduction in any medium, provided you give appropriate credit to the original author(s) and the source, provide a link to the Creative Commons license, and indicate if changes were made.

\section{References}

Bernier-Villamor L, Navarro E, Sevilla F, Lazaro JJ (2004) Cloning and characterization of a 2-Cys peroxiredoxin from Pisum sativum. J Exp Bot 55:2191-2199

Bogdan C, Rollinghoff M, Diefenbach A (2000) Reactive oxygen and reactive nitrogen intermediates in innate and specific immunity. Curr Opin Immunol 12:64-76

Castellanos-Martínez S, Gestal C (2013) Pathogens and immune response of cephalopods. J Exp Mar Biol Ecol 447:14-22

David E, Tanguy A, Moraga D (2007) Peroxiredoxin 6 gene: a new physiological and genetic indicator of multiple environmental stress response in Pacific oyster Crassostrea gigas. Aquat Toxicol 84:389-398

De Zoysa M, Ryu JH, Chung HC, Kim CH, Nikapitiya C, Oh C, Kim H, Saranya Revathy K, Whang I, Lee J (2012) Molecular characterization, immune responses and DNA protection activity of rock bream (Oplegnathus fasciatus), peroxiredoxin 6 (Prx6). Fish Shellfish Immunol 33:28-35

Gestal C, Castellanos-Martinez S (2015) Understanding the cephalopod immune system based on functional and molecular evidence. Fish Shellfish Immunol 46:120-130

Huang CF, Sun ZJ, Zhao YF, Chen XM, Jia J, Zhang WF (2011) Increased expression of peroxiredoxin 6 and cyclophilin A in squamous cell carcinoma of the tongue. Oral Dis 17:328-334

Jiang XM, Lu ZR, He HJ, Ye PL, Ying Z, Wang CL (2010) Effects of several ecological factors on the hatching of Sepiella maindroni wild and cultured eggs. Ying Yong Sheng Tai Xue Bao 21:1321-1326

Lee S, Jia B, Liu J, Pham BP, Kwak JM, Xuan YH, Cheong GW (2015) A 1-Cys peroxiredoxin from a thermophilic archaeon moonlights as a molecular chaperone to protect protein and DNA against stress-induced damage. PLoS One 10:e0125325

Livak KJ, Schmittgen TD (2001) Analysis of relative gene expression data using real-time quantitative PCR and the 2(-Delta Delta C(T)) Method. Methods 25:402-408

Manevich Y, Fisher AB (2005) Peroxiredoxin 6, a 1-Cys peroxiredoxin, functions in antioxidant defense and lung phospholipid metabolism. Free Radic Biol Med 38:1422-1432

Miller NJ, Rice-Evans C, Davies MJ, Gopinathan V, Milner A (1993) A novel method for measuring antioxidant capacity and its application to monitoring the antioxidant status in premature neonates. Clin Sci (Lond) 84:407-412

Mu C, Zhao J, Wang L, Song L, Zhang H, Li C, Qiu L, Gai Y (2009) Molecular cloning and characterization of peroxiredoxin 6 from Chinese mitten crab Eriocheir sinensis. Fish Shellfish Immunol 26:821-827

Nikapitiya C, De Zoysa M, Whang I, Kim CG, Lee YH, Kim SJ, Lee J (2009) Molecular cloning, characterization and expression analysis of peroxiredoxin 6 from disk abalone Haliotis discus discus and the antioxidant activity of its recombinant protein. Fish Shellfish Immunol 27:239-249

Park M, Shin HJ, Lee SY, Ahn TI (2005) Characterization of a cDNA of peroxiredoxin II responding to hydrogen peroxide and phagocytosis in Amoeba proteus. J Eukaryot Microbiol 52:223-230

Park H, Ahn IY, Kim H, Cheon J, Kim M (2008) Analysis of ESTs and expression of two peroxiredoxins in the thermally stressed Antarctic bivalve Laternula elliptica. Fish Shellfish Immunol 25:550-559

Poynton RA, Hampton MB (2014) Peroxiredoxins as biomarkers of oxidative stress. Biochim Biophys Acta 1840:906-912

Priyathilaka TT, Kim Y, Udayantha HM, Lee S, Herath HM, Lakmal HH, Elvitigala DA, Umasuthan N, Godahewa GI, Kang SI et al (2016) Identification and molecular characterization of peroxiredoxin 6 from Japanese eel (Anguilla japonica) revealing its potent antioxidant properties and putative immune relevancy. Fish Shellfish Immunol 51:291-302

Qiu L, Ma Z, Jiang S, Wang W, Zhou F, Huang J, Li J, Yang Q (2010) Molecular cloning and mRNA expression of peroxiredoxin gene in black tiger shrimp (Penaeus monodon). Mol Biol Rep 37:2821-2827

Rabilloud T, Berthier R, Vincon M, Ferbus D, Goubin G, Lawrence JJ (1995) Early events in erythroid differentiation: accumulation of the acidic peroxidoxin (PRP/TSA/NKEF-B). Biochem J 312(Pt 3):699-705

Robinson MW, Hutchinson AT, Dalton JP, Donnelly S (2010) Peroxiredoxin: a central player in immune modulation. Parasite Immunol 32:305-313

Schultz J, Milpetz F, Bork P, Ponting CP (1998) SMART, a simple modular architecture research tool: identification of signaling domains. Proc Natl Acad Sci USA 95:5857-5864

Shafer ME, Willson JA, Damjanovski S (2011) Expression analysis of the peroxiredoxin gene family during early development in Xenopus laevis. Gene Expr Patterns 11:511-516 
Tolomeo AM, Carraro A, Bakiu R, Toppo S, Place SP, Ferro D, Santovito G (2016) Peroxiredoxin 6 from the Antarctic emerald rockcod: molecular characterization of its response to warming. J Comp Physiol B 186:59-71

Vidal EA, Villanueva R, Andrade JP, Gleadall IG, Iglesias J, Koueta N, Rosas C, Segawa S, Grasse B, Franco-Santos RM et al (2014) Cephalopod culture: current status of main biological models and research priorities. Adv Mar Biol 67:1-98

Wang Q, Chen K, Yao Q, Zhao Y, Li Y, Shen H, Mu R (2008) Identification and characterization of a novel 1-Cys peroxiredoxin from silkworm, Bombyx mori. Comp Biochem Physiol B Biochem Mol Biol 149:176-182

Wei-Wei S, Chun-Lin W (2009) Genetic diversity of Sepiella maindroni in cultured and natural populations. Oceanol et Limnol Sin 40:590-595

Wei-Wei S, Chang-Kao M, Chu S, Yu-Yu Z, Ping-Ping Z, Chun-Lin W (2012) Expression screening and annotation of Sepiella maindroni ink sac cDNA library. J Genet 91:e103-e108

Wood ZA, Poole LB, Karplus PA (2003a) Peroxiredoxin evolution and the regulation of hydrogen peroxide signaling. Science 300:650-653

Wood ZA, Schroder E, Robin Harris J, Poole LB (2003b) Structure, mechanism and regulation of peroxiredoxins. Trends Biochem Sci 28:32-40

Yeh H-Y, Klesius P (2007) cDNA cloning, characterization, and expression analysis of channel catfish (Ictalurus punctatus Rafinesque, 1818) Peroxiredoxin 6 gene. Fish Physiol Biochem 33:233-239

Zhang Q, Li F, Zhang J, Wang B, Gao H, Huang B, Jiang H, Xiang J (2007) Molecular cloning, expression of a peroxiredoxin gene in Chinese shrimp Fenneropenaeus chinensis and the antioxidant activity of its recombinant protein. Mol Immunol 44:3501-3509

Zheng WJ, Hu YH, Zhang M, Sun L (2010) Analysis of the expression and antioxidative property of a peroxiredoxin 6 from Scophthalmus maximus. Fish Shellfish Immunol 29:305-311

Zhou Y, Kok KH, Chun AC, Wong CM, Wu HW, Lin MC, Fung PC, Kung H, Jin DY (2000) Mouse peroxiredoxin V is a thioredoxin peroxidase that inhibits p53-induced apoptosis. Biochem Biophys Res Commun 268:921-927

\section{Publisher's Note}

Springer Nature remains neutral with regard to jurisdictional claims in published maps and institutional affiliations. 